\title{
Integrated 256 cell photonic phase-change memory with 512-bit capacity
}

\author{
J. Feldmann, N. Youngblood, X. Li, C. D. Wright, H. Bhaskaran and W. H. P. Pernice
}

\begin{abstract}
All-optical nonvolatile memories enable storage of telecommunication data without detours through electronic circuitry. Phase-change materials provide the means to embed such memories within integrated optical circuits and thus allow combining waveguide devices for information processing with local data storage. Using this concept, we realize an all-photonic memory circuit capable of storing 512 bits of data in an array of nanoscale phase-change devices. We employ multilevel addressing and wavelength multiplexing of microring resonators to write and read a 16x16 greyscale image with 2-bit resolution entirely in the optical domain. Our approach holds promise for implementing scalable architectures for on-chip optical data storage with long data retention and ultrafast access times.
\end{abstract}

Index Terms-Nonvolatile memory, Multilevel data storage, Phase-change materials, Photonic integrated circuits.

\section{INTRODUCTION}

$\mathrm{P}$ HASE-change materials (PCMs) are attractive candidates for implementing next-generation memory devices because of outstanding optical and electrical properties [1]-[3]. For photonic memories, the large optical contrast between the crystalline and amorphous material phase enable realizing reconfigurable structures that can be conveniently manipulated with short laser pulses. This way the phase-state can be reversibly selected, thus providing nonvolatile response to tunable photonic building blocks. Importantly, the PCM devices operate at nanoscale dimensions and are thus compatible with waveguide devices. In nanophotonic circuits, such PCM elements can be conveniently manipulated in the evanescent near field [4], [5]. By tailoring read and write pulse shapes, desired phase states and thus desired optical properties can be selected. This way the transmission past the device can be modulated which allows for utilizing the PCM cell as an optical memory system. By controlling the phase state continuously, multilevel optical storage with up to 32 levels has been demonstrated in a single element [6].

Evanescent coupling allows combining elementary phasechange devices with complex nanophotonic circuits. This way the rich toolbox of integrated optics can be employed to

Manuscript received June 29, 2019. J. F. and W. H. P. P are with the Institute of Physics of the WWU Münster, Münster, Germany (corresponding author to provide phone: +49 (0)251 / 83 63957; e-mail: wolfram.pernice@ unimuenster.de). enhance the functionality of PCM optical memories. In particular, broad optical bandwidth available to photonic waveguides provides the means to perform wavelength division multiplexing (WDM) in integrated devices and thus selectively address desired devices on a chip [7]-[9]. By embedding PCM devices in optical cavities, individual cells can be addressed by selecting the wavelength. Using cavities with good quality factors, many such cavities can be connected to a common bus waveguide for convenient write and read access.

Here we employ optical cavities in the form of microring resonators to implement a WDM addressing scheme for writing and reading multiple PCM elements on chip. We realize a photonic memory in array format with $16 \times 16$ elements, connected to a single input waveguide. In each memory we inscribe a four-level scheme for storing data with 2-bit resolution. The optical random-access memory (ORAM) is written and read out in the telecommunication C-band fully in the photonic domain and avoids electro-optical conversions.

Differing from electrical implementations of phase-change memories[10]-[15], photonic data storage devices are still in their infancy[16]-[19]. Because of the high speed and bandwidth of optical signal processing and especially the capability of multiplexing signals, all-optical memories hold promise for very fast and efficient data storage. The approach presented in this work provides a convenient addressing scheme using wavelength multiplexing and employs phase-change materials as the data storage element giving rise to a nonvolatile storage removing the need of continuously refreshing the memory states.

\section{WORKING PRINCIPLE}

A sketch of the photonic matrix memory implemented in this work is shown in fig. 1 (a). The memory consists of $\mathrm{N}$ rows (groups of ring resonators) connected to a single input waveguide. Each row further contains $\mathrm{N}$ individual memory cells. Every memory cell (see fig. 1 (b)) is comprised of an input and an output waveguide, connected by two microring resonators and a waveguide with a PCM-patch on top. Part of the light travelling down the input waveguide is coupled to the first (lower) ring resonator and to the PCM-waveguide. After

N. Y., X. L. and H. B. are with the Department of Materials of the University of Oxford, Oxford, UK.

C. D. W. is with the Department of Engineering, University of Exeter, Exeter, UK. 
(a)

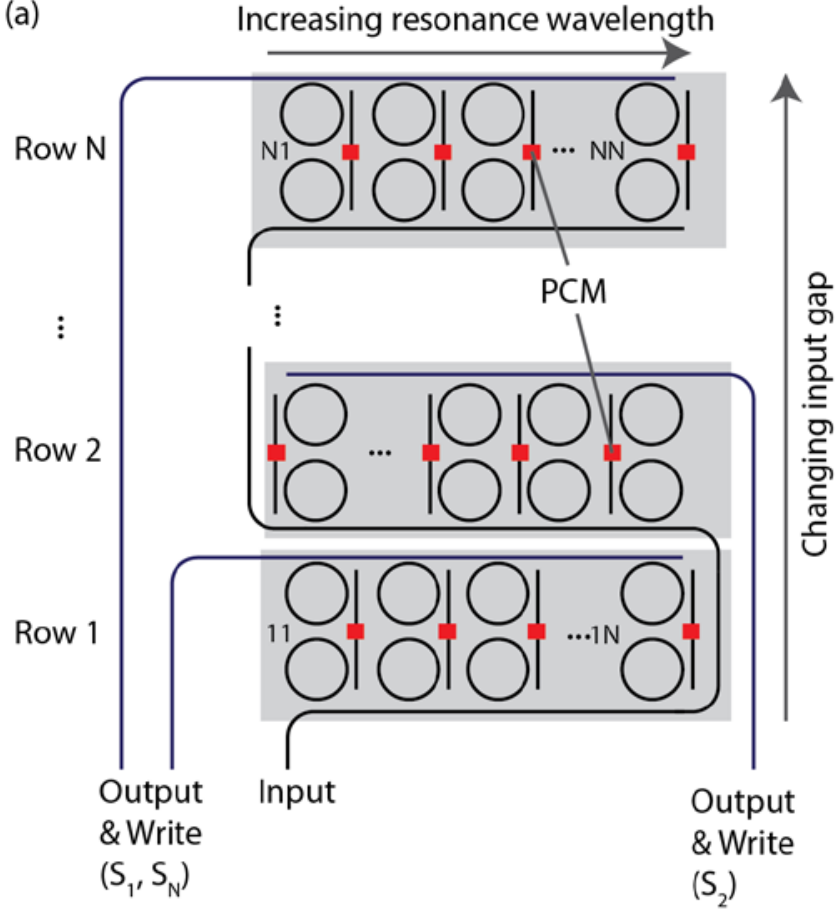

(b)

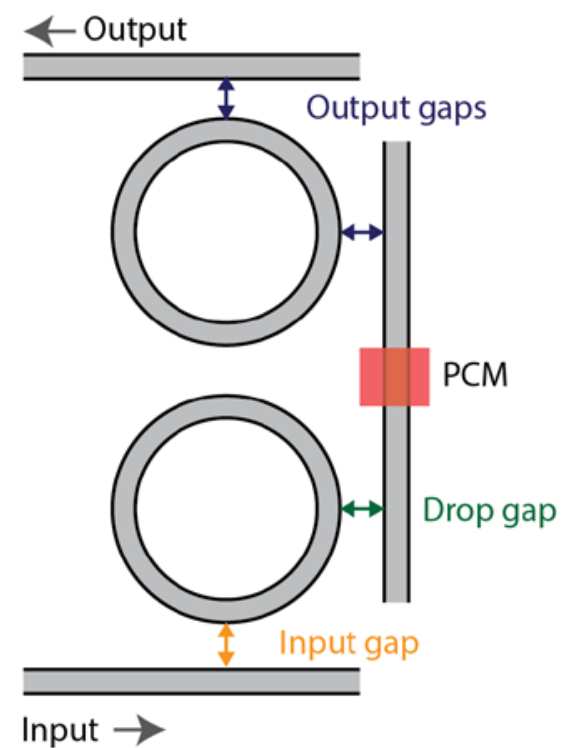

(c)

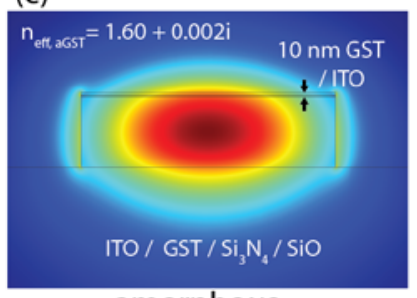

amorphous

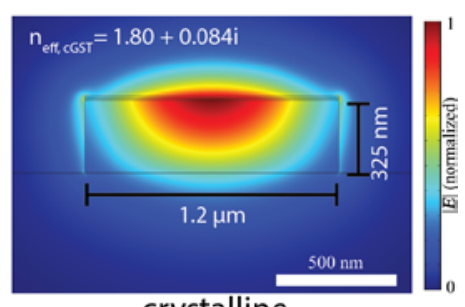

crystalline
Fig. 1. (a) Sketch describing the operation principle of the photonic memory. Several PCM-cells are combined to rows and can be addressed through wavelength division multiplexing. (b) Close up of a single memory cell indicating the important design parameters. (c) Finite element simulation of the optical mode profile with the PCM in the amorphous and crystalline state. Single mode waveguides with a height of $325 \mathrm{~nm}$ and a width of $1.2 \mu \mathrm{m}$ have been used in this work.

passing the phase-change cell the light is coupled to the output waveguide through a second ring resonator. By tuning the radius of the resonators, the wavelength from the input spectrum that is coupled to the PCM-cell can be adjusted. Both resonators (upper and lower in fig. 1 (b)) in a memory cell have the same radius and therefore the same resonance wavelengths and can this way multiplex and demultiplex the same input signal. Adjusting the coupling gaps between the resonators and waveguides in turn gives control over the amount of light that is coupled to the PCM- and output waveguide (as described in more detail later). For efficient readout, each row of memory cells receives the same amount of light which implies that the input light has to be equally split in $\mathrm{N}$ parts. The splitting ratio to the drop port of the first ring resonator (the PCM-waveguide) can be calculated with the following formula: $c_{e f f, i}=1 /(N+$ $1-i$ ), with ' $\mathrm{N}$ ' being the overall number of rows, and ' $\mathrm{i}$ ' the number of the row to be addressed. Each input gap (yellow) and drop gap (green) in a given row have the same values but are different for the individual rows to achieve equal splitting.

The output gaps (blue) are the same for all memory cells and are designed to couple all the light from the PCM-waveguide to the output waveguide. In this work a symmetric configuration with the same gap for input and drop port of the output resonator is used.

The phase-change cell deposited on top of the connection waveguide between the resonators is the actual data storage element. Light passing the PCM-cell is evanescently coupled to the memory cell (see Fig. 1 (c)). Because the imaginary part of the refractive index of the PCM strongly depends on its phase state, the incoming light is attenuated and thus the data is encoded in the transmission level of the memory cell. In the crystalline state most of the light passing a memory cell is absorbed in the PCM. In contrast, in the amorphous state, most of the light travels along the PCM waveguide without being attenuated because of the low absorption in the amorphous PCM. Depending on the ratio of crystalline and amorphous parts in the phase-change material, different levels of transmission can be obtained. Fig. 1 (c) shows the normalized electrical field obtained from finite element method (FEM) simulations (COMSOL Multiphysics). It can be seen that in the crystalline state, the optical mode is strongly pulled towards the PCM and the imaginary part of the effective refractive index is increased by more than an order of magnitude. In the crystalline phase this corresponds to an absorption of $2.96 \mathrm{~dB} / \mu \mathrm{m}$. The waveguide was designed to be single mode at a wavelength of $1550 \mathrm{~nm}$ with a height of $325 \mathrm{~nm}$ and a width of $1.2 \mu \mathrm{m}$.

Using the arrangement shown in Fig.1, readout of the individual cells is achieved in a two-dimensional scheme. The row can be selected by choosing the corresponding output port $\left(\mathrm{S}_{1}\right.$ to $\mathrm{S}_{\mathrm{N}}$ ) and the position of the cell inside a row can be selected via the wavelength, because all ring resonators in a row have slightly different resonance wavelengths. This way each memory cell can be individually addressed. It should be noted, that a configuration with two rings is used instead of depositing the PCM directly on top of a ring resonator, because the state of the PCM would highly influence the coupling to the resonator and also shift the resonance wavelength of the ring. This in turn would change the optical transmission to all following ring 
resonators of the same resonance wavelength and lead to incorrect readout results.

Writing data to a desired memory cell is achieved by coupling to the memory cell in the opposite direction. An optical pulse to program the PCM to a certain state is sent to the corresponding output port $\left(\mathrm{S}_{1}\right.$ to $\left.\mathrm{S}_{\mathrm{N}}\right)$. Again, by choosing the correct wavelength all PCM-cells can be addressed individually in this row. By sending the switching pulse only to a specific output port and not to the input port, we avoid that all cells with the same ring radius and resonance wavelength of the complete memory are switched.

\section{FABRICATION}

The photonic memory devices are fabricated using electronbeam lithography (EBL) with a Raith EBPG5150 system on silicon nitride-on-insulator substrates in three exposure steps. The layer stack consists of $325 \mathrm{~nm} \mathrm{Si}_{3} \mathrm{~N}_{4}$ on $3.3 \mu \mathrm{m} \mathrm{SiO}{ }_{2}$ deposited on a silicon carrier wafer. In a first lithography step windows for gold alignment markers are opened using the PMMA 950k 4.5 resist and development in IPA:MIBK (3:1) for 120 seconds. After depositing $7 \mathrm{~nm}$ of chromium and $70 \mathrm{~nm}$ of gold with a PVD and a subsequent lift-off in acetone, alignment markers for the next lithography steps are obtained. In a second step of lithography the photonic structures are exposed using $300 \mathrm{~nm}$ of the negative-tone e-beam resist AR-N 7520.12. After developing the mask and postbaking at $85^{\circ} \mathrm{C}$ for $60 \mathrm{~s}$, etching is carried out with a PlasmaPro 80 RIE (Oxford Instruments) with $\mathrm{CHF}_{3} / \mathrm{O}_{2}$ chemistry. The waveguides, which are designed to have a width of $1.2 \mu \mathrm{m}$, are fully etched into the silicon nitride waveguiding layer. After removing the remaining ebeam resist in oxygen Plasma for five minutes, the last lithography step to define the windows for the deposition of the phasechange material is performed in the same way as applied for the alignment markers. Finally, ten nanometers of the PCM $\mathrm{Ge}_{2} \mathrm{Sb}_{2} \mathrm{Te}_{5}$ (GST) and ten nanometer of indium tin oxide (ITO) only covering the GST are sputtered (5 mtorr working pressure, $15 \mathrm{sccm} \mathrm{Ar}, 30 \mathrm{~W}$ radio-frequency power, base pressure of $2 \times$ $10^{-6}$ torr). The ITO is applied as a protective layer for the PCM to avoid oxidation during the switching process and could also be replaced by other transparent materials, such as silicon dioxide. As the light couples evanescently to the PCM, the interaction with the material on top of the waveguide decreases exponentially with the distance so that the influence of the protective layer on the device performance can be neglected compared to the PCM After lifting off the PMMA in acetone, the photonic memory devices are obtained. Prior to characterizing the devices, the GST is crystallized on a hotplate at a temperature of $230^{\circ} \mathrm{C}$ for 15 minutes. Fig. 2 shows an optical micrograph of a fabricated device composed of 16 rows and 16 resonators per row, thus comprising 256 memory cells. The SEM image in Fig. 2 (b) shows a close up of a single memory cell after GST deposition and lift-off. The individual memory cells are composed of two ring resonators and a connection waveguide onto which the PCM element is deposited. We chose a double-ring configuration with connection waveguides in order to avoid that the resonance condition of the rings is affected by the phase change taking place in the memory element. It can be seen that the GST-patch is well aligned to the waveguide and the gaps between resonators and waveguides are open. In our devices, the length of the GST-patch along the waveguide is $3 \mu \mathrm{m}$ which provides sufficient optical contrast for memory readout. All input
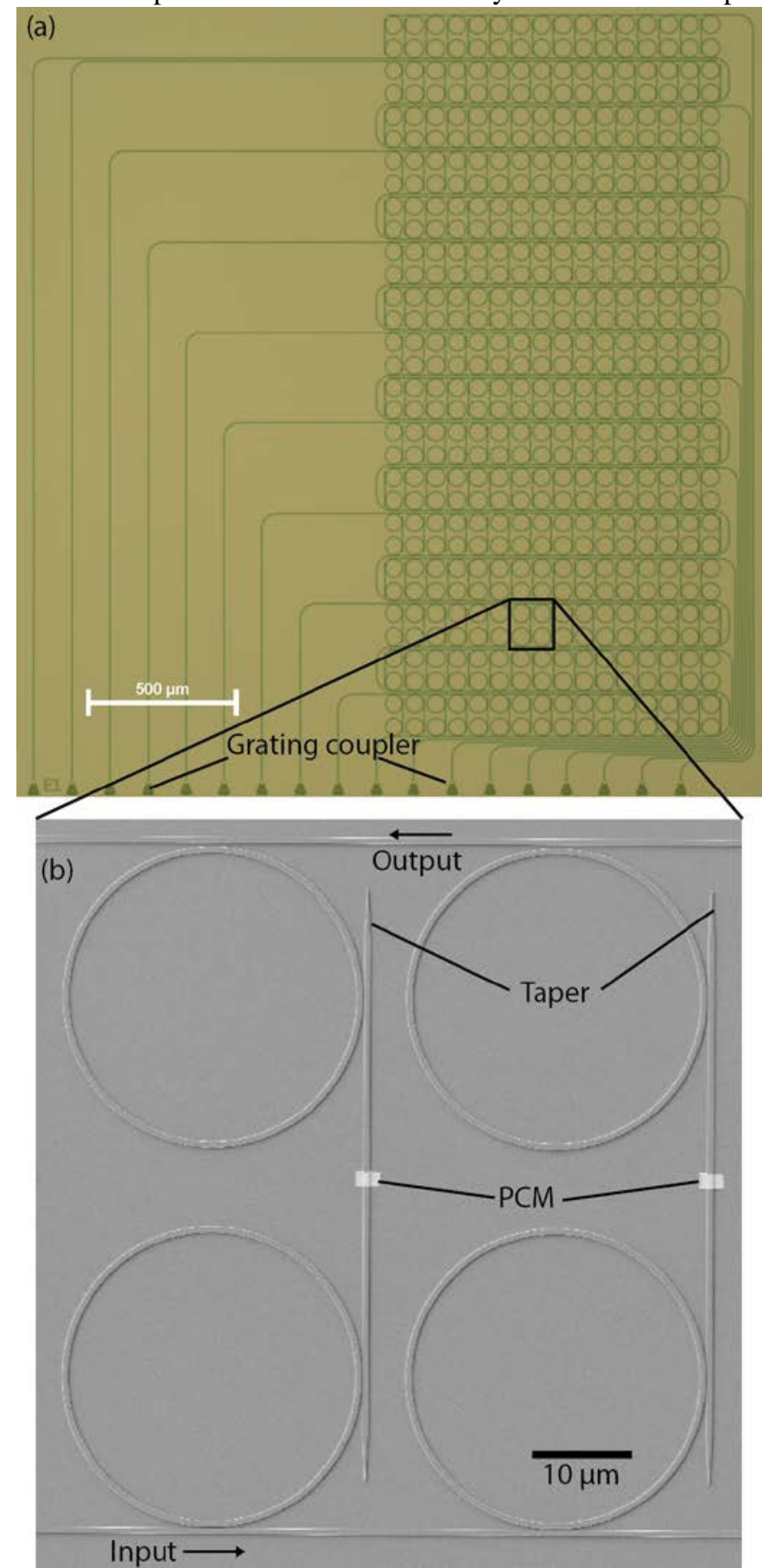

Fig. 2. (a) Optical micrograph of a photonic matrix memory with $16 \times 16$ memory cells. (b) Scanning-electron micrograph of a single memory cell within the array. The PCM element is placed on the drop waveguide in the two ring geometry in order to avoid resonance shifting upon phase change in the memory cell.

waveguides are terminated with focusing grating couplers in order to enable optical coupling to multiple input and output fibers. The connection waveguide between the rings shown in Fig.2b is tapered at both ends to avoid unwanted back reflections. 


\section{EXPERIMENTAL RESULTS}

\section{A. Measurement setup}

The experimental setup to characterize the memory devices is shown in Fig.3. The setup is comprised of two main parts, one for readout and one for write operations. The readout (red) is achieved using light from a tunable continuous-wave laser (Santec TSL 510), that is sent to the grating coupler at the input port of the device after adjusting the polarization for maximizing the coupling efficiency to the on-chip waveguides. A sufficiently low probe power is chosen (approx. $0.1 \mathrm{~mW}$ inside the input waveguide) in order not to influence the phase state of the memory cells [20]. After travelling through the onchip device, the transmitted light is collected from the output grating couplers with detectors $\mathrm{D}_{1}$ to $\mathrm{D}_{\mathrm{N}}$ (New Focus Model 2011). Optical circulators are used to separate the probe light from the "Set \& Reset" path (blue). For switching the phasechange material cells higher power optical pulses are generated from a second CW-laser (Santec TSL 710), by modulating the output power with an electro-optic modulator (EOM - Lucent Technologies, 2623CS), triggered by an electrical pulse generator (HP 8131A). An erbium-doped fiber amplifier (EDFA - Pritel) is employed to generate sufficient power for the set and reset operation of the individual PCM cells. The maximum pulse power used was 550 pJ for full recrystallization.

\section{B. Multiplexer}

Since the photonic memory employs wavelength division multiplexing (WDM) for addressing individual PCM cells in each row, in a first step the microring resonators have to be calibrated. The most important properties of a WDM device are the spectral position of the wavelength channels and their spectral separation. These parameters are determined by the resonance condition of the geometry of the ring resonators and can be tuned by adjusting their radii. From the resonance condition the relation for the difference in ring radii $\Delta r=$ $r_{1} \Delta \lambda / \lambda_{1}$ to obtain a certain wavelength shift can be derived with $r_{1}$ being the radius of the first resonator in a row, $\Delta \lambda$ the desired spacing between to resonances of different rings and the wavelength $\lambda_{1}$ of a resonance of the first resonator. In the design used in this work, the first ring resonator has a radius of $r_{1}=$ $30 \mu \mathrm{m}$, a desired spacing of the multiplexer $\Delta \lambda=800 \mathrm{pm}$ and a wavelength $\lambda_{1}=1550 \mathrm{~nm}$, leading to a radius difference between neighboring resonators of approximately $15 \mathrm{~nm}$.

Besides the ring radii, the most important design parameters are the gaps to the input port and the drop port for the first resonator as shown in fig. 1 (b). The gaps have to be fine-tuned in order to obtain equal splitting of the input light to all rows of the memory. Therefore, resonators with different configurations of the two parameters in the range from $20 \mathrm{~nm}$ to $800 \mathrm{~nm}$ are fabricated and the transmission to the through and the drop port is measured. The contour lines in fig. 3 (b) depict the measured splitting ratio between the two ports on resonance, whereas the colormap shows the corresponding loss of the device referenced to the off-resonance transmission at the through port. In an ideal resonator without any loss, the
TABLE I

MULTIPLEXER CONFIGURATION FOR AN ARRAY OF SIX ROWS

\begin{tabular}{llllll}
\hline \hline Row & $\begin{array}{c}\text { Input gap } \\
(\mathrm{nm})\end{array}$ & $\begin{array}{c}\text { Drop gap } \\
(\mathrm{nm})\end{array}$ & Ratio & $\begin{array}{c}\text { Loss } \\
\text { factor }\end{array}$ & Transmission \\
\hline 1 & 508 & 118 & 0,08 & 0,11 & 0,07 \\
2 & 605 & 345 & 0,11 & 0,20 & 0,07 \\
3 & 410 & 85 & 0,14 & 0,12 & 0,07 \\
4 & 410 & 150 & 0,21 & 0,20 & 0,07 \\
5 & 215 & 53 & 0,37 & 0,28 & 0,07 \\
6 & 53 & 53 & 0,98 & 0,40 & 0,07 \\
\hline \hline
\end{tabular}

maximum power transfer would always be achieved in a symmetric configuration in which the input gap is equal to the drop port gap [21], [22]. Because in the fabricated devices scattering loss, bending loss and absorption have to be taken into account, the configurations of maximum power transfer are slightly shifted to larger drop port gaps in the experimental data in agreement with theory. As can be easily seen from the

(a)
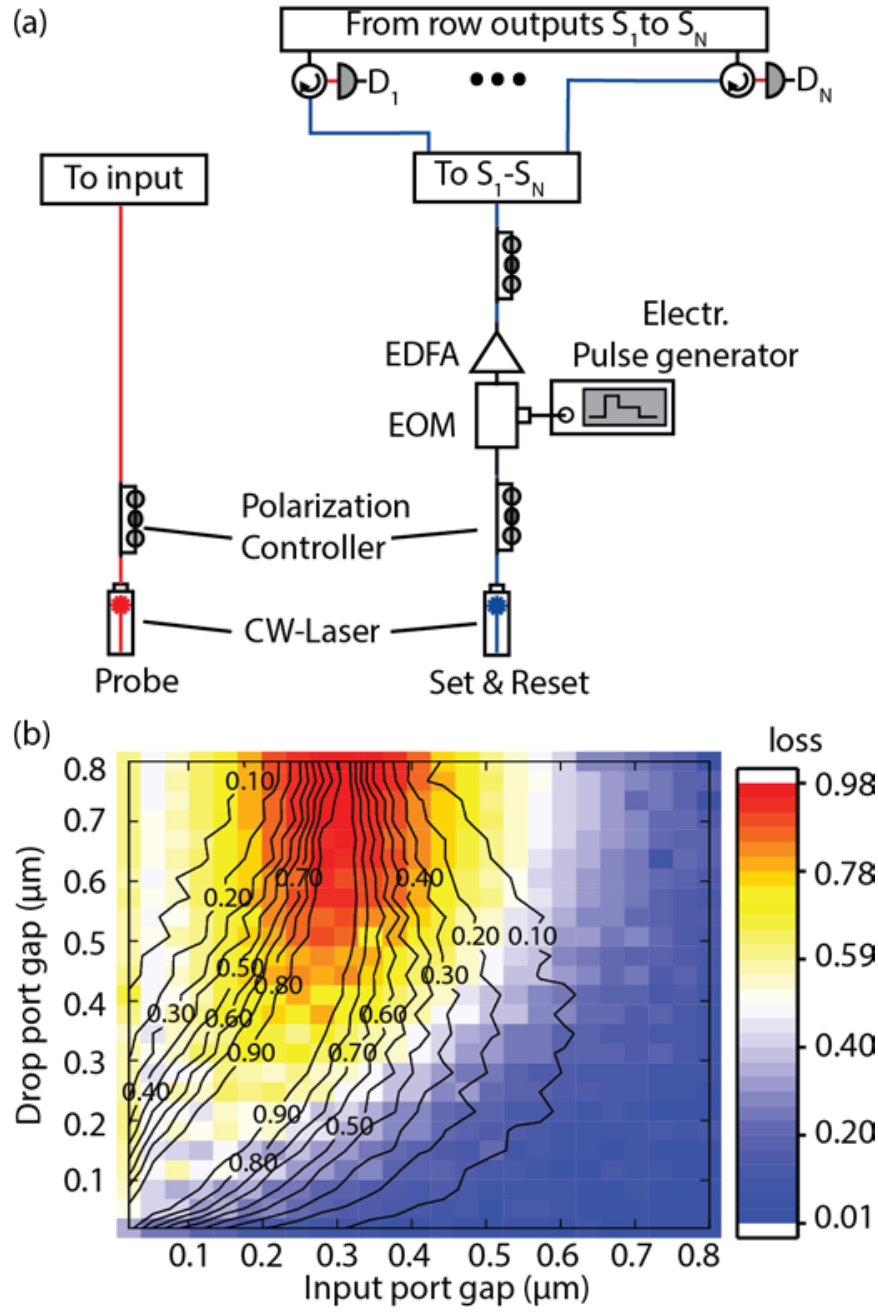

Fig. 3. (a) Experimental setup: a continuous-wave (CW) laser is used for readout with low power and sent to the input grating coupler of the photonic memory device. For (re-)setting the individual memory cells, optical pulses are cut from a CW-laser with an EOM, amplified and sent to the device. (b) Measured ratio between the output power at the through and drop port of a ring resonator in an add-drop configuration for different configurations of gaps. The contour lines depict the splitting ratio, the colormap illustrates the losses. 
colormap, a non-neglectable factor in choosing the gaps for equal splitting to all rows of the memory is the loss as there are for example configurations that couple $90 \%$ of the light to the drop port loosing almost no light but other configurations for the same ratio with loss near unity. The quality factors of the ring resonators are around 7.000, corresponding to a linewidth of roughly $220 \mathrm{pm}$.

Table I shows a resulting configuration for a memory array consisting of six rows. The experimentally obtained ratio $\left(T_{\text {drop }} /\left(T_{\text {drop }}+T_{\text {through }}\right)\right)$ and the loss factor are given. Based on these values the transmission to the PCM-cells in the row is calculated. It can be seen that with this approach all rows
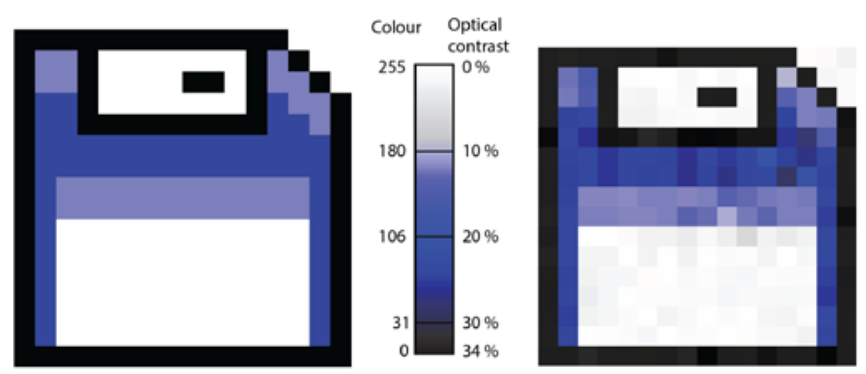

Fig. 5. Experimental demonstration of a 512-bit photonic memory. The drawing of a floppy disk on the left in two-bit resolution (four colors) is stored in an array of $16 \times 16=256$ memory cells.

receive seven percent of the incoming light. (Note that a lossfactor of 1 means that all light is scattered out and a loss factor of 0 describes no loss). The gaps between waveguide and resonator for the output ring resonator are selected for maximum power transfer to the output waveguide and were fixed to a symmetric configuration with gaps of $150 \mathrm{~nm}$ for all memory cells.

\section{Photonic memory}

Having designed a functional multiplexer and addressing scheme for the individual memory cells, in a next step the

(a)

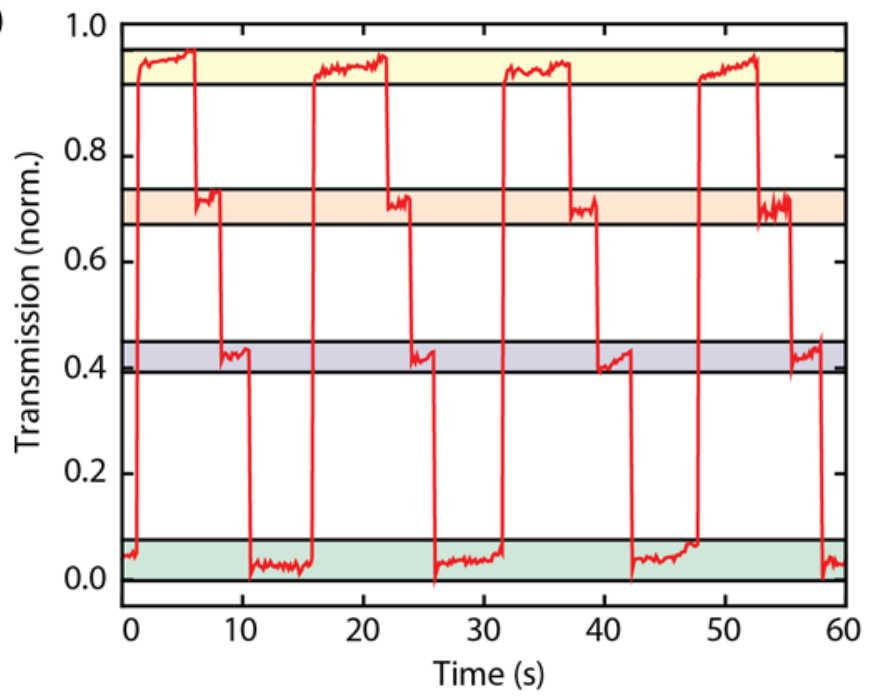

optical performance of a single PCM-cell is characterized in more detail. In order to increase the storage capability of our matrix memory, we employ multi-level bit storage in each memory cell. Fig. 4 (a) shows the optical transmission through a cell with a GST-patch of $3 \mu \mathrm{m}$ length measured as a function of time. By applying four different optical pulses, four distinguishable levels can be repeatedly set. This way in each memory cell 2-bit data can be recorded. The pulse shape used in the experiments consists of two rectangular pulses [6]. A first pulse of 50 ns length and approx. 200 pJ is used for amorphization (maximum optical transmission) immediately followed by a second pulse with lower energy and a width of 0 ns, 100 ns $(110 \mathrm{pJ}), 200 \mathrm{~ns}(230 \mathrm{pJ})$ and $300 \mathrm{~ns}(350 \mathrm{pJ})$ corresponding to the four different levels in fig. 4 (a). The second pulse is employed to keep the temperature of the PCM above the glass transition after the initial amorphization and induce crystallization. The longer the second pulse the more time is given for the PCM to recrystallize and lower transmission levels are achieved. Although a maximum pulse length of $350 \mathrm{~ns}$ is used in this experiment it should be noted that much shorter pulses can be applied to considerably reduce the switching times and energies [4], [23]. More details on the optical switching process can be found in our previous work [24]. PCMs are known to keep their phase-state for several years without applying additional power and the cycling endurance of phase-change materials has been shown to be at least $10^{12}$ when switched electrically [25] and $10^{6}$ in a similar waveguide configuration and switched optically as in this work [23]. Offering switching speeds in the sub-ns regime makes PCMs ideal candidates to be employed in optical randomaccess memories.

We design different multi-element memories by varying the number of rows and columns during photonic design. Fig. 4 (b) shows the measured transmission of a single row of a $6 \times 6$ photonic memory device in different states. The red line depicts the initial optical spectrum after fully crystallizing the phasechange material on a hotplate. The purple curve represents the

(b)

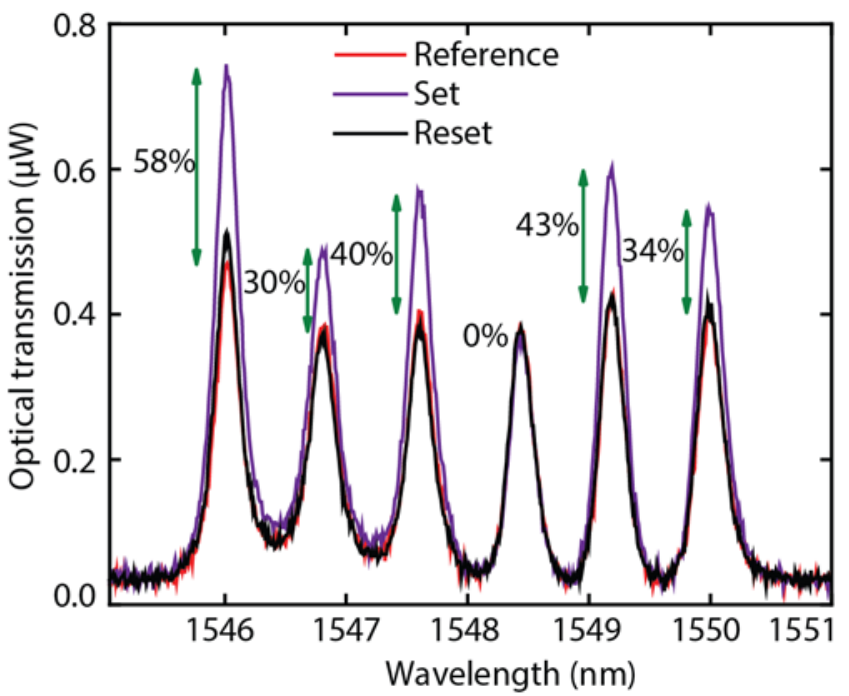

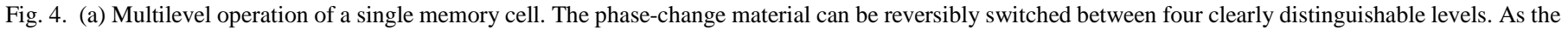

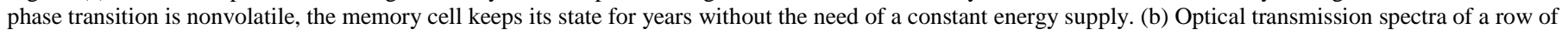
six memory elements. All PCM-cells can clearly be distinguished by their resonance wavelength and reversibly switched with optical pulses. 
"Set" state after data is stored, and the black line shows the transmission spectrum after resetting all elements employing optical pulses. It can be noticed that all six resonators operate on clearly separated resonance wavelengths with a spacing of approximately $800 \mathrm{pm}$ as defined in the design process for dense WDM compatibility. Higher channel densities could be achieved by using larger ring radii at the cost of increased device footprint. Reversible switching with optical pulses with a contrast of up to $58 \%$ is achieved in this experiment. All memory elements in this row can be addressed and set to a certain state individually without influencing the other PCMcells. For each memory element we inscribe 4 storage levels for 2-bit data capture, thus doubling the storage capability of the overall device. Since the optical contrast in the device is high and stable, our memory does not suffer from drift limitations as commonly found in electrical phase change memories.

In order to demonstrate scalability of the proposed photonic memory, we realize a 16x16 element device with 256 individual PCM elements as shown in Fig.2. Using the addressing scheme outlined above we record pixelated images into the photonic memory. A pictogram of a 16x16 pixel image illustrating a floppy disk (see fig. 5 left) is stored with two-bit resolution in a 16x16 memory cell array. Using 2-bit precision, four color values can be inscribed in each PCM cell. After readout as shown in Fig.5b all PCM-cells can be addressed individually using the WDM scheme, reproducing the desired pictogram with high accuracy. However, we note that not all resonance wavelengths in the memory for the same ring radii in different rows show spectral overlap due to fabrication imperfection. Therefore, in order to compensate for such wavelength offset, the probe and set wavelengths for the individual rows have to be adjusted accordingly. The overall device has a footprint of $1000 \times 2400 \mu^{2}$. It could be reduced by moving towards photonic materials with a higher refractive index, such as silicon[26], [27]. However, it should be noted that using smaller waveguide geometries also increases the requirements on the fabrication quality and precision in order to assure the correct resonance positions of the ring resonators. By inscribing higher number of intermediate levels as in our previous work, the color resolution can further be increased, thus increasing the overall storage density of the system.

\section{CONCLUSION}

We proposed and fabricated an all-optical non-volatile photonic random-access memory capable of storing up to 512 bits of data exploiting the high bandwidth inherent to optical signal processing. The demonstrated memory architecture with the two-dimensional addressing scheme can readily be scaled to larger storage capacities and gives rise to chip-integrated alloptical data storage for direct signal processing in the optical domain without electro-optic conversions. By employing wellestablished phase-change materials as the central memory element, the photonic ORAM offers fast and reversible switching in the sub-ns regime with high endurance and low energy consumption, as the PCM keeps its state at room temperature without the need of a constant bias energy.

\section{ACKNOWLEDGMENT}

The authors would like to acknowledge funding for this work from the European Union's Horizon 2020 research and innovation programme under grant agreement number 780848 (Fun-COMP). We further acknowledge funding through ERC consolidator grant 724707 (PINQS).s

\section{REFERENCES}

[1] M. Wuttig and N. Yamada, "Phase-change materials for rewriteable data storage,” Nat. Mater., vol. 6, no. 11, pp. 824-832, 2007.

[2] S. Raoux, F. Xiong, M. Wuttig, and E. Pop, "Phase change materials and phase change memory,” MRS Bull., vol. 39, no. 08, pp. 703-710, 2014.

[3] G. W. Burr et al., "Recent Progress in Phase-Change Memory Technology,” IEEE J. Emerg. Sel. Top. Circuits Syst., vol. 6, no. 2, pp. 146-162, 2016.

[4] C. Ríos et al., "Integrated all-photonic non-volatile multi-level memory,” Nat. Photonics, vol. 9, no. 11, pp. 725-732, 2015.

[5] J. Von Keitz et al., "Reconfigurable Nanophotonic Cavities with Nonvolatile Response,” ACS Photonics, vol. 5, no. 11, pp. 4644-4649, 2018.

[6] X. Li et al., "Fast and reliable storage using a 5 bit , nonvolatile photonic memory cell,” vol. 6, no. 1, 2019.

[7] A. Alduino and M. Paniccia, "Interconnects: Wiring electronics with light,” Nat. Photonics, vol. 1, no. 3, pp. 153-155, 2007.

[8] C. Sun et al., "Single-chip microprocessor that communicates directly using light,” Nature, vol. 528, no. 7583, pp. 534-538, 2015.

[9] J. Feldmann, N. Youngblood, C. D. Wright, H. Bhaskaran, and W. H. P. Pernice, "All-optical spiking neurosynaptic networks with self-learning capabilities,” Nature, vol. 569, no. 208, 2019.

[10] D. Loke et al., "Breaking the speed limits of phasechange memory.," Science, vol. 336, no. 6088, pp. 1566-9, 2012.

[11] A. Chen, “A review of emerging non-volatile memory (NVM) technologies and applications,” Solid. State. Electron., vol. 125, pp. 25-38, 2016.

[12] A. Pirovano, A. L. Lacaita, A. Benvenuti, F. Pellizzer, and R. Bez, "Electronic Switching in Phase-Change Memories,” IEEE Trans. Electron Devices, vol. 51, no. 3, pp. 452-459, 2004.

[13] H.-S. P. Wong et al., "Phase Change Memory,” Proc. IEEE, vol. 98, no. 12, pp. 2201-2227, 2010.

[14] G. W. Burr et al., "Phase change memory technology,” J. Vac. Sci., vol. 2004, no. 2, p. 903, 2010.

[15] R. E. Simpson et al., "Interfacial phase-change memory,” Nat. Nanotechnol., vol. 6, pp. 501-505, 2011.

[16] W. H. P. Pernice and H. Bhaskaran, "Photonic nonvolatile memories using phase change materials," Appl. Phys. Lett., vol. 101, no. 17, pp. 7-11, 2012.

[17] C. Rios, P. Hosseini, C. D. Wright, H. Bhaskaran, and W. H. P. Pernice, "On-chip photonic memory elements employing phase-change materials," Adv. 
Mater., vol. 26, no. 9, pp. 1372-1377, 2014.

[18] C. Rios et al., "Integrated all-photonic non-volatile multi-level memory,” Nat. Photonics, vol. 9, no. 11, pp. 725-732, 2015.

[19] M. Bagheri, M. Poot, M. Li, W. P. H. Pernice, and H. $\mathrm{X}$. Tang, "Dynamic manipulation of nanomechanical resonators in the high-amplitude regime and nonvolatile mechanical memory operation,” Nat.

Nanotechnol., vol. 6, no. 11, pp. 726-732, 2011.

[20] N. Youngblood et al., "Tunable Volatility of Ge2Sb2Te5 in Integrated Photonics,” Adv. Funct. Mater., vol. 29, 2019.

[21] A. Yariv and P. Yeh, "Photonics: Optical Electronics in Modern Communications.” Oxford University Press, p. 848, 2006.

[22] D. G. Rabus, Integrated Ring Resonators. SpringerVerlag Berlin Heidelberg, 2007.

[23] J. Feldmann et al., "Calculating with light using a chip-scale all-optical abacus,” Nat. Commun., vol. 8, 2017.

[24] C. Rios et al., "Mechanism for controlled switching of phase-change materials by evanescent-field coupling in integrated photonics,” vol. 8, no. 9, pp. 2-7, 2018.

[25] D. Kuzum, R. G. D. Jeyasingh, B. Lee, and H. P. Wong, "Nanoelectronic Programmable Synapses Based on Phase Change Materials for Brain-Inspired Computing,” Nano Lett., vol. 12, pp. 2179-2186, 2012.

[26] M. Rudé et al., "Optical switching at $1.55 \mu \mathrm{m}$ in silicon racetrack resonators using phase change materials,” Appl. Phys. Lett., vol. 103, no. 14, p. 141119, 2013.

[27] Q. Xu, B. Schmidt, J. Shakya, and M. Lipson, “Cascaded silicon micro-ring modulators for WDM optical interconnection,” Opt. Express, vol. 14, no. 20, p. $9431,2006$.

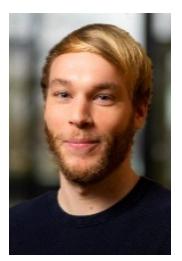

Johannes Feldmann received the B.S. degree (2013) and the M.S degree (2015) in Physics from the WWU Münster, Münster, Germany. He is currently pursuing the Ph.D. degree in Physics also at the Institute of Physics of the University of Münster.

His research is focused on the development of integrated nanophotonic circuits with phase-change materials for all-optical signal processing and computation.

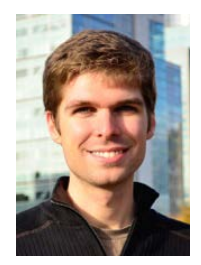

Nathan Youngblood received a $\mathrm{PhD}$ in Electrical Engineering from the University of Minnesota with research focusing on the integration of 2D materials with silicon photonics for optoelectronic applications. His research interests include integrated photonics, high-speed optoelectronics, novel computing methods, and nanoelectronics.

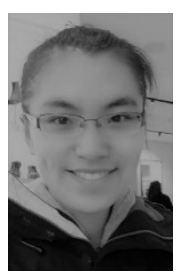

Xuan Li received the B.S. degree in electronic engineering from National University of Defense Technology, Changsha, China, in 2016. She is currently pursuing the Ph.D. degree in material science, University of Oxford, United Kingdom. From 2016.01 to 2016.06, she was an exchange student in Nanyang Technological University, Singapore. Her research interest includes photonic computation and storage with phase change materials.

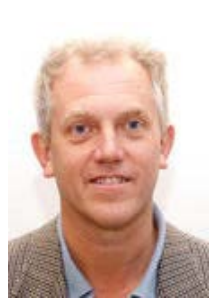

C. David Wright is Professor of Electronic and Computer Engineering at the University of Exeter, where he leads the NEST (Nano Engineering, Science and Technology) group. He received his BSc degree from Imperial College London, and his $\mathrm{PhD}$ from Manchester. Prior to moving to Exeter, he was a Reader in the School of Computer Science at the University of Manchester. He also worked as a Process Engineer for Philips Electronics. His main interests are in the area of chalcogenide and 2D materials photonics and electronics.

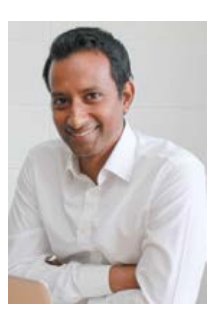

H. Bhaskaran is Professor of Applied Nanomaterials at the University of Oxford. He obtained his PhD from the University of Maryland, an MS degree also from Maryland, and a BE degree for the University of Pune. Before joining Oxford, he was at IBM Research Zurich, Yale University and the University of Exeter. An avid cricket and outdoors fan, he also carries out research on data storage and computing devices, nanomechanics and nanomanufacturing. He is also the Founding Director and Chief Scientific Officer of Bodle Technologies Limited.

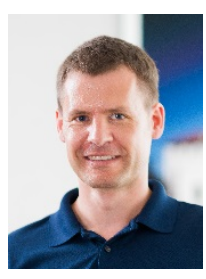

Wolfram Pernice received the Dipl. Ing. degree in Microsystems Technology from the University of Freiburg in 2004 and a PhD in Electrical Engineering from the University of Oxford in 2007. After postdoctoral training at Yale University, he joined the Karlsruhe Institute of Technology (KIT) in 2011 as an Emmy-Noether research group leader in 2011. Since 2015 he is a full professor of physics at the University of Münster. His research interests cover integrated quantum photonics, photonic neuromorphic architectures, as well as computational electrodynamics and on-chip non-linear optics. 